\title{
AUFSATZ
}

\section{Koalitionsaussagen der Parteien vor Wahlen. Eine Forschungsskizze im Kontext des deutschen Regierungssystems}

\author{
Frank Decker
}

Obwohl ihre politikwissenschaftlichen Ursprünge noch gar nicht so lange zurückreichen sie liegen in den 1960er Jahren - zählt die Koalitionsforschung heute zu den am intensivsten beackerten Themenfeldern der (vergleichenden) Regierungslehre. Dies gilt sowohl in theoretischer Hinsicht, wo sich ihre Modelle durch einen hohen Grad an Formalisierung auszeichnen, als auch empirisch. Detlef Noltes ${ }^{1}$ vor zwanzig Jahren formuliertes Diktum, wonach die Koalitionstheorie am Ende sei, hat sich als voreilig erwiesen. Die Kritik bezog sich auf die bescheidene Erklärungskraft der bis dahin vorherrschenden office- und policybezogenen Modelle der Koalitionsbildung, die - befördert durch die neo-institutionalistische Wende der Politikwissenschaft - seither jedoch überzeugend erweitert und fortgeschrieben wurden. ${ }^{2}$ Die Koalitionsforschung avancierte auf diese Weise zu einem Themenbereich von besonders hohem kumulativem Erkenntnisfortschritt. ${ }^{3}$

Mehr noch als die Ausstrahlungskraft des neo-institutionalistischen Paradigmas dürfte die Verbreiterung des empirischen Materials zu diesem Fortschritt beigetragen haben. Die veränderten Rahmenbedingungen des Regierens in den etablierten Demokratien und das Hinzutreten neuer demokratischer Systeme in Mittel- und Osteuropa nach 1989 erwiesen sich für die politikwissenschaftliche Komparatistik gleichermaßen als professioneller Glücksfall, boten sie ihr doch Gelegenheit, die vorhandenen Koalitionstheorien zu überprüfen und weiter zu entwickeln. Insbesondere die durch die Pluralisierung der Parteienlandschaften erhöhte Gefahr einer Destabilisierung der Regierungen hat das Bewusstsein von Neuem dafür geschärft, dass jegliche demokratische Politik ihrem Wesen nach Koalitionspolitik ist. Dies gilt für das Verhältnis der Parteien untereinander genauso wie im Binnenverhältnis der innerparteilichen Flügel und Gruppierungen.

Nimmt man beides zusammen, lassen sich insgesamt vier Ebenen der Koalitionsbildung und -politik unterscheiden: Erstens ist es das Ziel der Parteien, im Wettbewerb ein möglichst umfangreiches Bündnis verschiedener Wählergruppen zu bilden (elektorale Koalition). Ihr Erfolg hängt dabei - zweitens - mit davon ab, ob es gelingt, innerhalb der Partei

1 Vgl. Detlef Nolte, Ist die Koalitionstheorie am Ende? Eine Bilanz nach 25 Jahren Koalitionsforschung, in: PVS, 29. Jg. (1988), H. 2, S. $230-251$.

2 Umso merkwürdiger ist, dass in der deutschsprachigen lexikalischen Gesamtschau der Komparatistik - dem Band über die „westlichen Länder“ des Lexikons der Politik - der Begriff „Koalitionen“ unter immerhin 79 Einträgen keine Erwähnung findet. Manfred G. Schmidt (Hrsg.), Die westlichen Länder (Lexikon der Politik, Bd. 3, hrsg. von Dieter Nohlen), München 1992.

3 Vgl. Wolfgang C. Müller, Koalitionstheorien, in: Ludger Helms / Uwe Jun (Hrsg.), Politische Theorie und Regierungslehre, Frankfurt am Main / New York 2004, S. 267 - 301, S. 267. 
unterschiedliche, gruppenförmig organisierte Interessen zusammenzuführen und nach außen hin geschlossen zu vertreten (innerparteiliche Koalition). Im Außenverhältnis verbinden sich verschiedene Parteien zur Formulierung und Durchsetzung einer gemeinsamen Regierungspolitik. Hier muss - drittens - zwischen exekutiven und - viertens - legislativen Koalitionen unterschieden werden. In einer exekutiven Koalition bilden verschiedene Parteien zusammen die Regierung (im engeren Sinne), indem sie ein gemeinsames Programm vereinbaren und die Ressorts untereinander aufteilen. In einer legislativen Koalition stimmen sie gemeinsam über die Gesetze ab, in denen sich die Regierungspolitik materialisiert.

Im parlamentarischen Regierungssystem werden die legislativen durch die exekutiven Koalitionen weitgehend vorgezeichnet, während im präsidentiellen System beides tendenziell auseinanderfällt. Allerdings können sich die Regierungsformen in ihrer realen Funktionsweise durchaus angleichen. Auch die Mitwirkungsbefugnisse einer zweiten parlamentarischen Kammer führen dazu, dass auf der legislativen Ebene unter Umständen andere beziehungsweise breitere Koalitionen gebildet werden müssen als auf Regierungsebene. Von einem präsidentiellen System können Anreize ausgehen, gemeinsame Abstimmungskoalitionen schon vor den Wahlen anzubahnen (indem die Parteien sich durch Absprachen gegenseitig unterstützen). Wie die legislativen Mehrheiten zustande kommen, hängt also einerseits von den institutionellen Rahmenbedingungen der Koalitionspolitik ab und andererseits von ihrer politisch-kulturell vorgeprägten Praxis.

Während die Bildung elektoraler Koalitionen ausschließlich Thema der politologischen Wahl- und Parteienforschung geblieben ist, hält die Koalitionsforschung ihr Augenmerk ganz auf die exekutiven und legislativen Bündnisse gerichtet. Die innerparteilichen Strukturen finden dabei neuerdings ebenfalls Berücksichtigung, jedoch nur soweit sie für das Zustandekommen und Funktionieren der Koalitionen relevant sind. Dem liegt die Überlegung zugrunde, dass die Koalitionspräferenz einer Partei nicht von allen ihren Teilen gleichermaßen geteilt und unterstützt werden muss. ${ }^{4}$ Generell lässt sich in der neueren Literatur eine stärkere thematische Hinwendung zur Koalitionspolitik ausmachen, das heißt zur Frage, wie in Koalitionen regiert wird. ${ }^{5}$ Ausgangs- und Schwerpunkt der Forschung bleibt aber nach wie vor die Koalitionsbildung, auf der auch im Folgenden das Hauptaugenmerk liegt. $^{6}$

\section{Koalitionsaussagen: ein weißer Fleck in der Forschung}

Bei aller sonstigen Fülle der Literatur zur Koalitionsbildung fällt auf, dass das Thema „Koalitionsaussagen" dort bislang kaum eine Rolle gespielt hat. Erst in jüngster Zeit ist es Ge-

4 Vgl. Eric Linhart, Rationales Wählen als Reaktion auf Koalitionssignale am Beispiel der Bundestagswahl 2005, in: PVS, 48. Jg. (2007), H. 3, S. 461 - 484, S. 472.

5 Vgl. Sabine Kropp, Koalitionsregierungen, in: Oscar W. Gabriel / dies. (Hrsg.), Die EU-Staaten im Vergleich, Wiesbaden 2008, S. 514 - 549, S. 538 ff.

6 Dabei wird ausschließlich die erste Phase der Koalitionsbildung betrachtet, die sich auf das $\mathrm{Zu}-$ standekommen beziehungsweise die Anbahnung der Koalition erstreckt. Die inhaltlichen Koalitionsverhandlungen, die einen weiteren Theorieschwerpunkt bilden und in der Literatur zumeist im Rahmen von portfolio-Ansätzen untersucht werden, bleiben unberücksichtigt. Vgl. zum Beispiel Michael Laver / Kenneth A. Shepsle, Coalitions and Cabinet Government, in: American Political Science Review, 84. Jg. (1990), H. 3, S. $873-890$. 
genstand ausdrücklicher Forschungsbemühungen geworden. ${ }^{7}$ Man mag in der Klage über dieses Versäumnis eine deutsche Voreingenommenheit erkennen, nachdem die Frage, ob und mit wem sich die Parteien am liebsten zum Regieren zusammentun möchten, hierzulande inzwischen den Charakter eines politischen Gesellschaftsspiels angenommen hat. Die eigentliche inhaltliche Auseinandersetzung droht nicht nur in Wahlkampfzeiten hinter der leidigen „Koalitionsfrage“ immer mehr zu verblassen. Dennoch (oder gerade deshalb) ist es verwunderlich, dass sich auch deutsche Politologen nicht bemüßigt gefühlt haben, Koalitionsaussagen in ihre einschlägigen Überblicksdarstellungen wenigstens aufzunehmen. ${ }^{8}$ Das Versäumnis lässt sich auch am Fehlen eines begrifflichen Pendants zur „Koalitionsaussage“ im Englischen ablesen, wo der in Rede stehende Sachverhalt nur scheinbar genauer als „pre-electoral coalition“ umschrieben wird.

Tatsächlich hat sich die Forschung für das, was mit Blick auf die Koalitionen vor den Wahlen passiert, bislang nur im Rahmen der gängigen office- und policy-seeking-Theorien der Koalitionsbildung interessiert. Diese besagen, dass die Parteien die Bündnisse zum einen aus Gründen des Machterwerbs und der Übernahme von Regierungsämtern schließen, zum anderen um ihre politikinhaltlichen Ziele durchzusetzen. Beide Erklärungsansätze verhalten sich komplementär zueinander. So wie die Erlangung von Regierungsmacht Voraussetzung ist, um die Ziele zu erreichen, so darf das Regieren umgekehrt nicht zum bloßen Selbstzweck erstarren, wenn die Legitimität des demokratischen Parteienwettbewerbs gewahrt und die Macht selbst gesichert werden soll. Diese Erkenntnis des gesunden Menschenverstandes konnte in empirischen Analysen wiederholt bestätigt werden. Der gemeinsame Beitrag des office- und policy-seeking nimmt sich allerdings nicht sonderlich eindrucksvoll aus. Nach Sabine Kropp, Suzanne Schüttemeyer und Roland Sturm ${ }^{9}$ kann damit maximal die Hälfte der Varianz der Koalitionsbildung in den west- und osteuropäischen Demokratien erklärt werden. Die Ausblendung der Koalitionsaussagen mutet vor diesem Hintergrund merkwürdig an. Die Vertreter der office- und policy-seeking-Theorien unterstellen offenbar, dass sich die Parteien nur zu jenen Koalitionen vorab bekennen, die sie aus ideologischen oder Machterwerbsgründen ohnehin geschlossen hätten. Dass die Koalitionsaussagen von den office- oder policy-bezogenen Erklärungen abweichen und sie darüber einen eigenständigen Einfluss auf die Koalitionsbildung erlangen könnten, kommt ihnen offenbar nicht in den Sinn.

Sona Golder ${ }^{10}$ nennt zwei weitere Gründe, die es nahelegen, sich mit Koalitionsaussagen zu beschäftigen. Der erste liegt in der schlichten Verbreitung. Ein Viertel bis ein Drittel der von ihr untersuchten Regierungen in 22 etablierten Demokratien von 1946 bis 1998 basierten danach auf prä-elektoralen Koalitionen. Fragt man, ob im Vorfeld der Wahlen von mindestens einer Partei eine Koalitionsaussage gemacht wurde, liegt der Anteil mit 44 Prozent noch höher. ${ }^{11}$ Die Verteilung zwischen den Staaten weist dabei allerdings große Unter-

7 Vgl. Sona Nadenichek Golder, Pre-electoral Coalitions in Comparative Perspective: A Test of Existing Hypotheses, in: Electoral Studies, 24. Jg. (2005), H. 4, S. 643 - 663, S. 644.

8 Vgl. Wolfgang C. Müller, a.a.O. (Fn. 3); Sabine Kropp, a.a.O. (Fn. 5).

9 Vgl. Sabine Kropp / Suzanne S. Schüttemeyer / Roland Sturm, Koalitionen in West- und Osteuropa. Theoretische Überlegungen und Systematisierung des Vergleichs, in: dies. (Hrsg.), Koalitionen in West- und Osteuropa, Opladen 2002, S. 7-40, S. 10.

10 Vgl. Sona Nadenichek Golder, a.a.O. (Fn. 7), S. 645 f.

11 Vgl. Sona Nadenichek Golder, Pre-Electoral Coalition Formation in Parliamentary Democracies, in: British Journal of Political Science, 36. Jg. (2006), H. 2, S. 193 -212, S. 194. 
schiede auf. In einigen Ländern stellen Koalitionsaussagen den Regelfall dar (unter anderem Deutschland, Frankreich, Österreich, Spanien), in anderen bilden sie die Ausnahme (Dänemark, Niederlande, Schweden).

Der zweite Grund ist ein normativer. Koalitionsaussagen sind aus demokratischer Sicht begrüßenswert, denn sie erhöhen den in Vielparteiensystemen reduzierten Einfluss des Wählers auf die Regierungsbildung. In einem System multipler Koalitionsmöglichkeiten entscheiden ja letztlich nicht die Bürger über die Allianzen, sondern die Parteien beziehungsweise genauer: die Parteiführungen. Die Rede vom Wähler als angeblichem „Souverän“, an Wahlabenden regelmäßig zu hören, kommt vor diesem Hintergrund einer Verhöhnung gleich. Indem sie die Zahl der tatsächlich ins Auge gefassten gegenüber den theoretisch möglichen Alternativen verringern, sorgen Koalitionsaussagen für mehr Klarheit und verhindern, dass der Wähler die sprichwörtliche „Katze im Sack“ kauft. ${ }^{12}$ Golder ${ }^{13}$ hält sie deshalb für ein probates Mittel, die jeweils besten Elemente der Mehrheits- und Konsensdemokratie miteinander zu verbinden.

\section{Begriff und Kategorisierung von Koalitionsaussagen}

Die Vernachlässigung des Themas Koalitionsaussagen in der Forschung rührt zu einem Gutteil auch aus der begrifflichen Unsicherheit, was unter dem Phänomen genau zu verstehen ist. Golder ${ }^{14}$ legt ihrer Definition der „prä-elektoralen Koalition“ zwei Kriterien zugrunde: Erstens dürfen die Parteien nicht völlig unabhängig voneinander und gegeneinander im Wettbewerb stehen; sie stimmen sich also in ihrer Wahlkampfführung ab. Und zweitens müssen die abgestimmten Strategien vor der Wählerschaft offengelegt werden. Die drei begrifflichen Elemente, die in dieser Definition enthalten sind - Abhängigkeit, Koordination und Offenheit -, laden allesamt zu Missverständnissen ein. Dass die Parteien im Wahlkampf nicht unabhängig voneinander agieren, gilt als generelle Feststellung auch ohne Koalitionsaussagen. ${ }^{15}$ Das Wesen des demokratischen Parteienwettbewerbs liegt in der Interaktion, also im Reagieren auf die Positionen der anderen. Ist das Kriterium der Abhängigkeit / Unabhängigkeit von daher zu weit gefasst, so bedeutet es aber eine begriffliche Verengung, wenn ein koordiniertes Vorgehen im Wahlkampf als Merkmal von Koalitionsaussagen betrachtet wird. Damit könnte der Eindruck entstehen, dass Verhandlungen zwischen den prospektiven Koalitionspartnern bereits vor den Wahlen geführt werden. Unter bestimmten institutionellen Umständen mag das tatsächlich so sein; doch handelt es sich hier keineswegs um den Regelfall.

Ähnlich problematisch ist das Kriterium der Offenheit. Golder möchte damit jene Vorab-Koalitionen aus der Betrachtung ausschließen, die lediglich implizit, also nicht ausdrücklich angekündigt werden, so wie es der deutsche Begriff Koalitionsaussage suggeriert.

12 Eckhard Jesse, Koalitionsaussagen der Parteien vor Bundestagswahlen, in: ders. I Eckart Klein (Hrsg.), Das Parteiensystem im wiedervereinigten Deutschland, Berlin 2007, S. 85 - 96, S. 96.

13 Vgl. Sona Nadenichek Golder, a.a.O. (Fn.11), S. 211.

14 Vgl. ebenda, S. 195.

15 Dies bedeutet zugleich, dass die Koalitionsaussagen selber ein potenzieller Gegenstand der wettbewerblichen Auseinandersetzung sind. So kann zum Beispiel ein nicht gegebenes Koalitionssignal von dem zurückgewiesenen Partner kritisiert oder die Glaubwürdigkeit einer Koalitionsaussage von den Mitbewerbern öffentlichkeitswirksam in Zweifel gezogen werden. 
Darin liegt ebenfalls eine nicht nachvollziehbare Verkürzung. Will man auch die nicht ausdrücklich erklärten Koalitionspräferenzen in die Analyse einbeziehen, würde sich statt „Koalitionsaussage“ der weiter gefasste Begriff „Koalitionssignal“ anbieten. ${ }^{16}$

Als erstes Analysekriterium wäre demnach die Stärke des Koalitionssignals zu nennen. Die möglichen Ausprägungen reichen hier von ausdrücklichen und eindeutigen Festlegungen auf lediglich einen potenziellen Partner (unter ebenso eindeutigem Ausschluss aller anderen), die von der Partei geschlossen vertreten werden ${ }^{17}$, über verklausuliert formulierte beziehungsweise aus öffentlichen Äußerungen und / oder vergangenem Verhalten verlässlich ableitbare Präferenzen, die auch mehrere Partner gleichzeitig einschließen können, bis hin zu neutralen oder unverbindlichen Stellungnahmen („demokratische Parteien müssen untereinander koalitionsfähig sein“), die eine Präferenz nicht erkennen lassen und deshalb auch nicht als Koalitionssignal gewertet werden können.

Als zweites muss zwischen positiven und negativen Koalitionssignalen unterschieden werden. Erstere besagen, dass man eine Koalition mit einem bestimmten Partner (oder mehreren) bilden möchte, letztere schließen Koalitionen mit einem bestimmten Partner (oder mehreren) aus.

Drittens schließlich ist zu fragen, ob es sich um symmetrische Koalitionssignale handelt, die von dem (den) umworbenen Partner(n) in derselben Intensität erwidert werden, oder um asymmetrische, also einseitige Signale. Starke Asymmetrie liegt vor, wenn positive und negative Koalitionssignale aufeinanderprallen, der eine Partner also eine Koalition bilden möchte und der andere nicht.

Fasst man die drei Kriterien zusammen, indem man (mit positivem oder negativem Vorzeichen versehene) Indices bildet, lassen sich die Koalitionssignale in einer Matrix darstellen. Für die deutsche Bundestagswahl 2005 würde eine solche Matrix, die alle relevanten, also für die Koalitionsbildung theoretisch vorstellbaren Partner einschließen sollte, wie folgt aussehen:

\begin{tabular}{|c|c|c|c|c|c|}
\hline & $\mathrm{CDU} / \mathrm{CSU}$ & SPD & FDP & Grüne & Linke \\
\hline CDU/CSU & - & 0 & +2 & $0^{*}$ & -2 \\
\hline SPD & 0 & - & $0^{*}$ & $+1^{*}$ & $-2^{*}$ \\
\hline FDP & +2 & $-2^{*}$ & - & $-2^{*}$ & -2 \\
\hline Grüne & $-1^{*}$ & $+2^{*}$ & $0^{*}$ & - & $-1^{*}$ \\
\hline Linke & -2 & $+1^{*}$ & -2 & $+1^{*}$ & - \\
\hline \multicolumn{6}{|c|}{$\begin{array}{l}\text { Anmerkung: Die „Zeilenpartei“ macht eine Aussage zur „Spaltenpartei“. } 0 \text { steht für kein erkennbares, } 1 \\
\text { für ein implizites und } 2 \text { für ein ausdrückliches Koalitionssignal. Das Vorzeichen macht deutlich, ob es } \\
\text { sich um ein positives oder negatives Koalitionssignal handelt. Die asymmetrischen Koalitionssignale sind } \\
\text { mit Sternchen versehen. } \\
\text { Quelle: Darstellung in Anlehnung an Eric Linhart, a.a.O. (Fn. 4), S. } 472 \text { f. }\end{array}$} \\
\hline
\end{tabular}

16 So zum Beispiel Franz Urban Pappi / Alexander Herzog / Ralf Schmitt, Koalitionssignale und die Kombination von Erst- und Zweitstimme bei den Bundestagswahlen 1953 bis 2005, in: ZParl, 37. Jg. (2006), H. 3, S. $493-513$.

17 Linhart betrachtet die innerparteiliche Geschlossenheit / Uneinigkeit bezüglich der Koalitionssignale als eigenständiges Kriterium. Hier wird es unter die Stärke des Koalitionssignals subsumiert, da eine innerparteilich umstrittene Koalitionsaussage kaum als eindeutig oder sicher verlässlich gewertet werden kann. Eric Linhart, a.a.O. (Fn. 4), S. 472. 
Auf der Basis einer solchen Matrix lassen sich Aussagen über die wahrscheinliche Koalitionsbildung treffen. ${ }^{18}$ Da eine Zusammenarbeit mit der Linkspartei von allen anderen Parteien abgelehnt wurde, war davon auszugehen, dass diese im Koalitionspoker keine Rolle spielen würde. Ausgeschlossen werden konnte auch eine Zusammenarbeit von FDP und Rot-Grün im Rahmen einer Ampelkoalition, da diese Möglichkeit von den Freidemokraten vorab klar verworfen wurde. Etwas komplizierter liegt der Fall der Jamaika-Koalition. Eine Zusammenarbeit von Union und Grünen hätte den impliziten Koalitionssignalen zumindest der Grünen widersprochen und das Zusammengehen der FDP mit den Grünen sogar den offenen Wortbruch der Liberalen erfordert. Die Aussicht auf eine bürgerliche Dominanz in der Koalition hätte die FDP nach der Wahl vielleicht dazu verführen können, einen solchen Wortbruch zu begehen - immerhin war die Union ihr erklärter Wunschpartner. So aber blieben als politisch mögliche Bündnisse nur Schwarz-Gelb, Rot-Grün und die Große Koalition. Die Wahrscheinlichkeit einer Neuauflage von Rot-Grün war dabei mit Blick auf die zu erwartenden Stimmenanteile der beiden Parteien äußerst gering. Weil es am Ende für die bürgerliche Mehrheit rechnerisch nicht langte, blieb als Auffanglösung nur noch die Große Koalition.

\section{Institutionelle und politisch-kulturelle Erklärungsfaktoren}

Bei der Frage nach institutionellen und politisch-kulturellen Faktoren der Koalitionspolitik muss analytisch zwischen den Faktoren unterschieden werden, die der Koalitionsbildung im Allgemeinen zugrunde liegen, und den (Teil-)Faktoren, die speziell die Koalitionssignale beeinflussen. Was die Koalitionsbildung im Allgemeinen angeht, konnte die Vorhersagekraft der office- und policy-bezogenen Theorien durch die zusätzliche Berücksichtigung institutioneller Faktoren wesentlich verbessert werden. Dies gilt zumal, wenn der Institutionenbegriff im weiteren Sinne ausgelegt wird, so dass er nicht nur die „harten“ Variablen der Verfassungsstruktur mit einschließt, sondern auch die „weichen“ der Verfassungspraxis. Letztere hängen wiederum von den historischen Prägungen der Politischen Kultur ab, die insofern als eigenständiger Faktor ebenfalls berücksichtigt werden müssen.

Die office- und policy-bezogenen Erklärungsmodelle der formalen Koalitionstheorien lassen sich unmittelbar aus den Strukturen des Parteiensystems ableiten. Diese werden sowohl von gesellschaftlichen als auch institutionellen Variablen geprägt, die aber zum Teil auch unabhängig auf die Koalitionsbildung einwirken. Als institutionelle Faktoren sind zu nennen: (1) die Regierungsform, (2) die territoriale Staatsorganisation, (3) das Wahlsystem und (4) die Konflikt- beziehungsweise Konsenskultur. Es versteht sich von selbst, dass zahlreiche Wechselbeziehungen bestehen, die in die Analyse einbezogen werden müssen.

\subsection{Regierungsform}

Auf die unterschiedliche Logik der Koalitionsbildung im Parlamentarismus und Präsidentialismus wurde bereits hingewiesen. Parlamentarische Systeme, die durch die institutionelle 
Verschmelzung der Regierung mit der sie tragenden Parlamentsmehrheit charakterisiert sind, bevorzugen exekutive Koalitionen, während im gewaltentrennenden Präsidentialismus legislative Koalitionen vorherrschen. Anderweitige institutionelle Systemmerkmale und politisch-kulturelle Dispositionen können aber dazu führen, dass die Systeme in der Praxis ähnlich funktionieren. So schließen zum Beispiel festgefügte exekutive Koalitionen in den parlamentarischen Systemen ein Regieren mit wechselnden legislativen Mehrheiten nicht aus, wie der in den skandinavischen Ländern verbreitete Minderheitsparlamentarismus beweist. ${ }^{19}$ Umgekehrt kann in den präsidentiellen Systemen nicht davon ausgegangen werden, dass sich die politischen Akteure Konstellationen eines divided government regelrecht herbeisehnen. Bemerkenswert ist auch, dass das Land mit der am stärksten festgefügten exekutiven Koalition überhaupt - die Schweiz mit ihrer immerwährenden Konkordanzregierung - systemlogisch zu den präsidentiellen Regierungsformen gehört. ${ }^{20}$

Unterhalb oder jenseits der groben Unterteilung in Systemtypen müssen deshalb mit Blick auf die Koalitionsbildung weitere institutionelle Merkmale berücksichtigt werden. Bei den parlamentarischen Systemen gilt das zum Beispiel für die Rolle, die das Staatsoberhaupt im Verfahren der Regierungsbestellung sowie im Gesetzgebungsprozesss spielt. In den parlamentarischen Republiken, wo die Präsidenten direkt vom Volk oder durch eine Versammlung bestellt werden, ist die Wahl selbst koalitionspolitisch bedeutsam, indem sie eine potenzielle Signalfunktion für die Parlamentswahlen ausübt. So sind zum Beispiel in Deutschland Koalitionsentscheidungen gelegentlich durch die Bundespräsidentenwahl vorweggenommen worden.

Des Weiteren kann den parlamentarischen Mehrheitserfordernissen bei der Investiturabstimmung oder förmlichen Regierungswahl Bedeutung zukommen. ${ }^{21}$ Genauso wichtig sind die ungeschriebenen Regeln der Mehrheitsbildung. Gilt zum Beispiel das Prinzip, dass der Regierungsauftrag stets bei der stärksten Partei liegt und diese in einer Koalition automatisch das Amt des Regierungschefs für sich reklamieren kann, sind die Anreize, VorabKoalitionen zu bilden, geringer, weil die Zahl der möglichen Koalitionsformate von vornherein begrenzt ist. Noch größer dürfte der Einfluss der Bestellungsregeln im Präsidentialismus sein. Da hier sowohl die Exekutivspitze als auch das Parlament vom Volk gewählt werden, sind die Übergänge zum Faktor Wahlrecht fließend. Dies gilt besonders für die Mehrheitserfordernisse, während man die zeitliche Terminierung der Wahlen, also die Frage, ob die Parlamentswahlen unmittelbar nach den Präsidentschaftswahlen, parallel zu diesen oder inmitten der Wahlperiode des Präsidenten stattfinden, eher der Regierungsform zuordnen müsste.

\subsection{Territoriale Staatsorganisation}

In föderalen Systemen beeinflussen sich die Koalitionsbildungen auf den beiden staatlichen Ebenen wechselseitig. Der Grad der Beeinflussung hängt zum einen davon ab, wie stark die

19 Vgl. Sabine Kropp / Suzanne S. Schüttemeyer / Roland Sturm, a.a.O. (Fn. 9), S. 34 f.

20 Vgl. Winfried Steffani, Artikel „Parlamentarisches und präsidentielles Regierungssystem“, in: Manfred G. Schmidt (Hrsg.), a.a.O. (Fn. 2), S. 288 - 294, S. 292.

21 Vgl. Michael Laver / Ian Budge (Hrsg.), Party Policy and Coalition Government, London 1992, S. $11 \mathrm{f}$. 
Ebenen institutionell miteinander verschränkt sind. Im dualföderalistischen System der USA ist die Verschränkung traditionell gering, so dass dem Senat hier zwar in struktureller, aber nicht in funktioneller Hinsicht die Rolle eines föderativen Vertretungsorgans zukommt. Im verbundföderalen System der Bundesrepublik Deutschland gewinnen die Mehrheitsverhältnisse in der Zweiten Kammer dagegen maßgebliche Bedeutung für die Regierungsfähigkeit. Hier führt die notorische Überlagerung von föderalen und parteipolitischen Konflikten dazu, dass die Koalitionsbildungen in den Ländern ganz oder überwiegend aus Perspektive der Bundespolitik betrachtet werden. Auf der einen Seite dient die Landespolitik als Labor, um neue Koalitionen auf der Bundesebene zu testen oder „serienreif“ zu machen. Auf der anderen Seite entwickeln Regierung und Opposition im Bund ein Interesse, die Koalitionen auf der Landesebene möglichst gleich zu halten. ${ }^{22}$ Beides übt auf die Parteien einen starken Druck aus, sich koalitionspolitisch zu bekennen. ${ }^{23}$

Der Grad der wechselseitigen Beeinflussung der Koalitionsbildung wird auch von der Identität der Regierungsformen auf den beiden staatlichen Ebenen bestimmt. Weisen die Regierungssysteme der Gliedstaaten sowohl untereinander als auch im Verhältnis zum Gesamtstaat nur geringe institutionelle Unterschiede auf, wie etwa in Deutschland, beeinflussen sich die Koalitionsbildungen stärker, als es bei abweichenden institutionellen Strukturen der Fall ist. ${ }^{24}$ Letzteres gilt zum Beispiel für Österreich mit seinen Proporzregierungen in den Bundesländern oder die Schweiz, wo die Kantonsregierungen im Unterschied zur Bundesregierung in direkter Volkswahl bestellt werden.

\subsection{Wahlsystem}

Der stärkste unmittelbare Einfluss auf die Koalitionsbildung geht in institutioneller Hinsicht vom Wahlsystem aus. Die Haupttrennlinie verläuft dabei nicht, wie man im Anschluss an eine gängige Lehrbuchweisheit vermuten könnte, zwischen Mehrheits- und Verhältniswahl, sondern zwischen der relativen Mehrheitswahl und allen übrigen Systemen. Nur die relativen Mehrheitswahlsysteme bringen mit stabiler Verlässlichkeit Zweiparteienstrukturen auf der parlamentarischen Ebene hervor, die die Bildung von „einfarbigen“ Regierungen im Regelfall gestatten. Für die absolute Mehrheitswahl französischer Provenienz gilt dagegen, dass sie aufgrund der 50-Prozent-Schwelle mit einem Mehrparteiensystem kompatibel bleibt, obwohl sie einen größeren Disproportionalitätsgrad aufweist als die Systeme der relativen

22 Vgl. Uwe Jun, Koalitionsbildung in den deutschen Bundesländern. Theoretische Betrachtungen, Dokumentation und Analyse der Koalitionsbildung auf Länderebene seit 1949, Opladen 1994, S. 93 ff. Dass die aktuellen oder absehbaren Mehrheitsverhältnisse im Bundesrat auf die Koalitionsbildung im Bund nennenswerten Einfluss nehmen, wird man hingegen nicht behaupten können. Dies dürfte in erster Linie darauf zurückzuführen sein, dass die Bundesregierungen bei ihrem Amtsantritt bisher noch nie mit einer gegnerischen Mehrheit im Bundesrat konfrontiert waren.

23 In personeller Hinsicht macht sich dieser Bekenntnisdruck allerdings nicht bemerkbar. Hier hat die starke Verflechtung zwischen Bundes- und Landespolitik zu der verbreiteten Unsitte geführt, dass Landespolitiker auf vorderen Listenplätzen ihrer Parteien zu den Bundestagswahlen kandidieren, später dann aber auf das gewonnene Mandat verzichten.

24 Vgl. Frank Decker, Die Regierungssysteme in den Ländern, in: ders. (Hrsg.), Föderalismus an der Wegscheide?, Wiesbaden 2004, S. 169 - 201. 
Mehrheitswahl. ${ }^{25}$ Der Koalitionszwang, der von der absoluten Mehrheit ausgeht, ist sogar stärker als bei den Verhältniswahlsystemen, da er sich auch auf die elektorale Ebene erstreckt. Die Parteien müssen Absprachen über gemeinsame Kandidaten treffen, was nur bei entsprechenden politischen und / oder personellen Zusicherungen „funktioniert“. Die absolute Mehrheitswahl führt also dazu, dass die Koalitionsverhandlungen zum Teil bereits im Vorfeld der Wahl beziehungsweise während des Wahlverfahrens stattfinden und nicht erst danach. ${ }^{26}$ Ein ähnlicher Effekt geht von Präferenzstimmensystemen aus (übertragbare Einzelstimme).

Auch im Rahmen der Verhältniswahl können bestimmte Regelungen zu Absprachen herausfordern. Das Bundestagswahlrecht mit seiner Kombination von Fünfprozenthürde, Grundmandatsklausel ${ }^{27}$ und Zweistimmensystem bietet dafür ein gutes Beispiel. Für die Koalitionsbildung kommt vor allem den Sperrklauseln große Bedeutung zu, die im Interesse der Regierungsstabilität einer zu starken Zersplitterung des Parteiensystems vorbeugen sollen. Sperrklauseln befördern das taktische Wählen. Droht eine Partei, die für die Regierungsbildung benötigt wird, unter fünf Prozent zu bleiben, könnten Koalitionswähler sie bewusst über die Hürde hieven. In der Bundesrepublik wird dieser Effekt durch die Möglichkeit des Stimmensplittings verstärkt. Fordert eine Partei die Wähler mit einer gezielten Zweitstimmenkampagne dazu auf, tut sie das nach aller Erfahrung auf der Basis einer klaren Koalitionspräferenz. ${ }^{28}$

\subsection{Konflikt- beziehungsweise Konsenskultur}

Bei den weichen Faktoren besteht immer die Gefahr, dass sie als bloße Verlegenheits- oder Restkategorie für das herhalten müssen, was sich mit den härteren (und das heißt in der Regel auch: leichter messbaren) Variablen der Koalitionsbildung - institutionelle Regeln, office- und policy-seeking - nicht erklären lässt. Dies kann aber kein Grund sein, sie außer Acht zu lassen. Systeme mit vergleichbaren institutionellen Strukturen können, wie man weiß, in der Praxis sehr unterschiedlich „ticken“. Eine Schlüsselrolle spielt hierbei das Parteiensystem, das wiederum ein Produkt historisch geronnener gesellschaftlicher Konfliktlinien (cleavages) darstellt. Auch wo sie auf ökonomische Interessen abstellen, bedürfen die Konfliktlinien stets der kulturellen Überhöhung, um parteibildend wirksam zu werden. ${ }^{29}$

$25 \mathrm{Vgl}$. Arend Lijphart, Patterns of Democracy. Government Forms and Performance in Thirty-Six Countries, New Haven / London 1999, S. 162. Genau aus diesem Grund - weil es die Vorzüge der einfacheren Regierungsbildung mit der größeren Legitimität einer Vielparteienstruktur verbindet - wird es von Kritikern des bestehenden Verhältniswahlsystems in der Bundesrepublik als Alternative zum britischen Modell gerne ins Spiel gebracht. Vgl. zum Beispiel Roman Herzog, Strukturmängel der Verfassung? Erfahrungen mit dem Grundgesetz, München 2000, S. 69 ff.

26 Vgl. Roman Herzog, a.a.O. (Fn. 25), S. 78 f.

27 Die Grundmandatsklausel bietet eine Möglichkeit, die Fünfprozenthürde zu umgehen. Parteien können danach im Verhältnis ihres Zweitstimmenanteils in das Parlament einziehen, wenn sie mindestens drei Wahlkreise gewinnen. In den 1950er Jahren machte sich die Union diese auf ihr Betreiben eingeführte Bestimmung zunutze, indem sie den für die Koalitionsbildung benötigten bürgerlichen Brückenparteien durch den Verzicht auf eigene Kandidaten freiwillig die Direktmandate überließ.

28 Vgl. Franz Urban Pappi / Alexander Herzog / RalfSchmitt, a.a.O. (Fn. 16).

29 Vgl. Karl Rohe, Wahlen und Wählertraditionen in Deutschland. Kulturelle Grundlagen deutscher Parteien und Parteiensysteme im 19. und 20. Jahrhundert, Frankfurt am Main 1992, S. 23 f. 
Deshalb ist es schwierig, den allgemeinen Einfluss der Politischen Kultur auf die Regierungsweise eines Landes von den kulturellen Prägungen seines Parteiensystems analytisch zu separieren (siehe Abbildung 1).

Politische Kulturen lassen sich nach dem Grad ihrer Konsens- oder Konfliktorientierung unterscheiden. Gesellschaftliche und politisch-institutionelle Ebene müssen dabei auseinander gehalten werden. Länder, die infolge von ethnischen, konfessionellen oder sozialökonomischen Konflikten stark polarisiert sind, können sich auf der politisch-institutionellen Ebene entscheiden, diese Polarisierung auszutragen (wie Frankreich oder Italien) oder sie durch Konsensbildung zu überwinden. Letzteres kann historisch später oder früher erfolgen, wie ein Vergleich zwischen Österreich und der Schweiz zeigt, und in unterschiedlicher Form geschehen. In Österreich wurde zum Beispiel die Große Koalition als Standardregierungsmodell etabliert, während die von ihrer institutionellen Verfasstheit her vergleichbaren parlamentarischen Systeme Dänemarks und Schwedens Minderheitsregierungen den Vorzug gegeben haben. In Deutschland setzte sich unterdessen unter ebenfalls vergleichbaren Bedingungen ein antagonistisches Modell des Parteienwettbewerbs durch, in dem Große Koalitionen allenfalls Notlösungen sind und Minderheitsregierungen als Sündenfall des parlamentarischen Systems generell abgelehnt werden. ${ }^{30}$

Das Konsensmodell zeichnet sich gegenüber dem Wettbewerbsmodell einerseits durch eine größere Regelhaftigkeit beim Regierungsauftrag, andererseits aber auch durch eine größere Flexibilität aus. Beides macht Koalitionssignale bis zu einem gewissen Grade entbehrlich. Indem er das Parlament als Ganzes gegenüber der Regierung aufwertet, nimmt der skandinavische Minderheitsparlamentarismus den Wahlen etwas von der schicksalhaften „Richtungsentscheidung“, die ihnen anderswo zugeschrieben wird. Die Erwartung, dass Wählerstimmen und Parlamentssitze in Regierungseinfluss umgesetzt werden sollen, wird an alle Parteien herangetragen, nicht nur jene der Regierung. ${ }^{31}$

Die Bedeutung des Faktors Kultur lässt sich zudem am Umgang mit einmal gegebenen Koalitionsversprechen ablesen. Die moralisch hoch aufgeladenen Verratsvorwürfe, die der FDP und ihrem Vorsitzenden Hans-Dietrich Genscher nach der Aufkündigung der sozialliberalen Koalition 1982 entgegenschlugen, wären in einer lateinisch-katholischen Kultur wie Frankreich oder Italien, wo das Finassieren zum politischen Alltagsgeschäft gehört und allenfalls zynische Reaktionen hervorruft, kaum vorstellbar gewesen - in der protestantisch geprägten Bekenntnis- und Schuldkultur der Bundesrepublik sollten sie noch lange nachhallen. ${ }^{32}$ Auch die Rigorosität, mit der die deutsche Öffentlichkeit den Wortbruch der hessischen SPD-Politikerin Andrea Ypsilanti quittierte, als diese nach der Landtagswahl eine Zusammenarbeit mit der Linken entgegen ihrer vorherigen Ankündigung doch nicht mehr ausschließen wollte, würde in manch anderem Land befremdlich wirken.

Der vergleichende Überblick macht deutlich, wie sehr auch die Koalitionsbildung in institutioneller und politisch-kultureller Hinsicht der Pfadabhängigkeit unterliegt. Langfristig gilt das vor allem in Bezug auf die bevorzugten Regierungs- und Koalitionsformate, während

30 Vgl. Marc Debus, Pre-Electoral Alliances, Coalition Rejections, and Multiparty Governments, Baden-Baden 2007, S. 26 ff.

31 John Fitzmaurice, Divided Government. The Case of Denmark, in: Robert Elgie (Hrsg.), Divided Government in Comparative Perspective, Oxford / New York 2001, S. 146 - 166, S. 165.

32 Vgl. Hartmut Soell, Helmut Schmidt. Macht und Verantwortung - 1969 bis heute, München 2008, S. 892. 


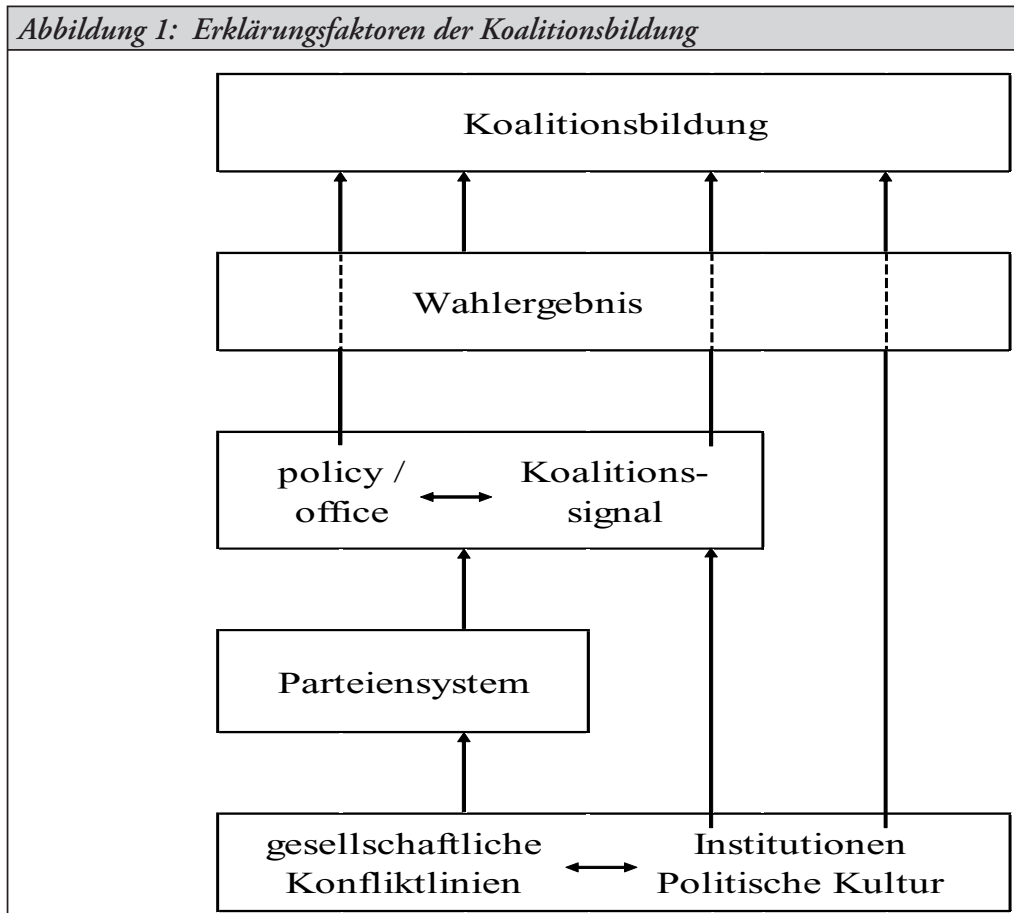

Quelle: Eigene Darstellung.

ein kurzfristiger Primat des Etablierten bei der parteipolitischen Zusammensetzung der Bündnisse besteht: Amtierende Koalitionen tendieren dazu, sich selbst zu erneuern, wenn die Mehrheitsverhältnisse es erlauben. ${ }^{33}$ Damit wird zugleich die Bedeutung des Akteursverhaltens unterstrichen. So wie an den Weggabelungen der europäischen Parteiensysteme unterschiedliche Koalitionen der herrschenden Eliten mit bestimmten gesellschaftlichen Gruppen stehen, entfalten auch die einmal getroffenen Koalitionsentscheidungen auf der Regierungsebene eine hohe Bestandskraft. In Westdeutschland war es zum Beispiel keineswegs ausgemacht, dass sich die kleine Koalition nach 1949 als Standardformat im Bund durchsetzen würde, nachdem in den Ländern zu dieser Zeit noch Große Koalitionen und Allparteienregierungen die Regel waren. ${ }^{34}$ Erst Konrad Adenauers bewusste Entscheidung für eine bürgerliche Koalition (die von der SPD unter Kurt Schumacher mit einem kompromisslosen Oppositionskurs beantwortet wurde) ebnete dem gegnerschaftlichen Modell des Parteienwettbewerbs den Weg, das in der Folge auch in den Ländern Schule machte.

33 Regierungs- und Koalitionswechsel finden im parlamentarischen System fast genauso oft während der Legislaturperiode statt wie im Zuge einer Wahl. Letzteres ist immer dann der Fall, wenn veränderte Mehrheitsverhältnisse eine neue Koalition ermöglichen oder erzwingen. In den übrigen Fällen werden die Koalitionsparteien sich hüten, dem Noch-Partner schon vor der Wahl eine Absage zu erteilen, würden sie damit doch ihre eigene Regierungsarbeit dementieren. Vgl. Klaus von Beyme, Artikel „Regierungswechsel“, in: Manfred G. Schmidt (Hrsg.), a.a.O. (Fn. 2), S. 401 405.

34 Vgl. Frank Decker, a.a.O. (Fn. 24), S. 186 ff. 


\section{Auswirkungen von Koalitionssignalen auf Wählerverhalten und Parteistrategien}

Aus Sicht des Wählers sind die Koalitionssignale in jedem Falle von Vorteil, geben sie ihm doch mehr Gewissheit, welche Regierungskonstellationen aus der Wahl hervorgehen können. Damit hat seine Stimme einen größeren unmittelbaren Einfluss auf die Regierungsbildung. Dies dürfte vor allem in den mehrheitsdemokratisch geprägten Systemen als legitimationsfördernd empfunden werden. Aus Sicht der Parteien könnten sich die Koalitionssignale dagegen als nachteilig erweisen, wenn sich die Wähler gerade wegen des Signals von der Partei abwenden. Im ungünstigsten Fall würde das dazu führen, dass die angestrebte Mehrheit verfehlt wird und die Wunschkoalition nicht zustande kommt. Die Parteien werden Koalitionssignale sicher nicht allein deshalb abgeben, weil dies einer allgemeinen Erwartungshaltung der Wählerschaft entspricht. Hinzukommen muss ihr eigenes Kalkül, dass das Signal von den Wählern tatsächlich honoriert wird. Hat sie dieses nicht, wird sie auf eine Koalitionsaussage vermutlich verzichten.

Dass verlässliche Auskünfte darüber, wie Koalitionssignale sich auf das Wahlverhalten auswirken, von der Wahlforschung nicht zu erlangen sind, liegt in der Natur der kontrafaktischen Fragestellung begründet. Es soll ja ein faktisches Ereignis (Wahlverhalten bei vorhandenem Koalitionssignal oder Wahlverhalten bei nicht vorhandenem Koalitionssignal) mit einem hypothetischen Ereignis verglichen werden, das genau die gegenteilige Konstellation unterstellt (Wahlverhalten bei nicht vorhandenem Koalitionssignal oder Wahlverhalten bei vorhandenem Koalitionssignal). Darüber hinaus hängt das Wahlverhalten nicht nur vom möglichen Koalitionssignal derjenigen Partei ab, die man favorisiert, sondern muss im Kontext aller Koalitionssignale betrachtet werden. In der empirischen Umsetzung birgt das nahezu unlösbare Probleme.

Entsprechend rar sind die in der Forschung hierzu bislang vorliegenden Studien. Sie arbeiten entweder mit experimentellen Ansätzen ${ }^{35}$ oder basieren auf räumlichen Modellen. ${ }^{36}$ Die Ergebnisse weisen dabei nicht sehr weit über das hinaus, was einem auch die Intuition sagt. So zeigen zum Beispiel Thomas Gschwend und Marc Hooghe ${ }^{37}$, dass die Anhänger kleiner Parteien bei missliebigen Koalitionssignalen in der Tendenz eher abtrünnig sind als die Anhänger großer Parteien. Wenig überraschend ist auch der Befund, wonach die generelle Akzeptanz oder Missbilligung des Koalitionspartners für das Verbleiben oder Abwandern eine wichtige Rolle spielt - unabhängig von den ideologischen Positionen, die dieser einnimmt. Eric Linhart versucht mit seinem Modell zu überprüfen, ob Union, FDP und SPD mit ihren Koalitionssignalen bei der Bundestagswahl 2005 gut beraten waren. „War es für Union und FDP strategisch sinnvoll, vor einer Koalition zwischen SPD, Grünen und Linkspartei zu warnen oder nicht? War es für die SPD und die CDU/CSU geschickt, eine große Koalition tendenziell eher abzulehnen oder wäre eine tendenzielle Zustimmung besser für sie gewesen?" 38 Die erste Frage wird mit einem klaren Ja beantwortet, während die

35 Vgl. Robert E. Goodin / Werner Güth / Rupert Sausgruber, When to Coalesce: Early Versus Late Coalition Announcement in an Experimental Democracy, in: British Journal of Political Science, 38. Jg. (2007), H. 1, S. 181 - 191; Thomas Gschwend / Marc Hooghe, Should I Stay or Should I Go? An Experimental Study on Voter Responses to Pre-electoral Coalitions, in: European Journal of Political Research, 47. Jg. (2008), H. 5, S. $556-577$.

36 Vgl. Eric Linhart, a.a.O. (Fn. 4).

37 Vgl. Thomas Gschwend/ Marc Hooghe, a.a.O. (Fn. 35), S. 574.

38 Eric Linhart, a.a.O. (Fn. 4), S. 482. 
zweite Frage für Union und SPD zu unterschiedlichen Ergebnissen führt. Die Union tat danach Recht daran, die Große Koalition eher auszuschließen, wohingegen es für die SPD besser gewesen wäre, sie weniger strikt auszuschließen. Auch das ist mit Blick auf die bis kurz vor der Wahl erhobenen Umfragewerte wenig erstaunlich, denn diese gaben der SPD praktisch keine Chance, ihre Regierungsmacht außerhalb einer Großen Koalition zu verteidigen.

Indirekten Aufschluss über die Rückwirkungen von (gegebenen oder nicht gegebenen) Koalitionssignalen auf das Wahlverhalten können auch Wanderungsanalysen und die Abfrage von Koalitionspräferenzen der Parteianhänger geben. Eine solche Analyse hätte die FDP zum Beispiel davor gewarnt, bei der Bundestagswahl 2002 ohne klare Koalitionsaussage zugunsten der Union ins Rennen zu gehen - was der überwiegenden Erwartungshaltung ihrer Anhänger widersprach. Nachdem sie ihren Fehler 2005 korrigierten, konnten die Liberalen prompt kräftig zulegen. Beleg für die Wirksamkeit ihrer Koalitionsaussage war dabei, dass im Vergleich zu 2002 ein größerer Teil der Wähler von der Möglichkeit des Stimmensplittings Gebrauch machte. ${ }^{39}$

Sind die Parteien mit Blick auf die erwartbare Wählerentscheidung gehalten, Koalitionssignale zu senden, stellt sich die Frage nach den Folgen. Welche Rückwirkungen hat ein Koalitionssignal auf die eigene inhaltliche Positionierung? Passen die Parteien ihre Programme - gleichsam in vorauseilendem Gehorsam - schon vorab dem gewünschten Ergebnis der Regierungsbildung an, indem sie sich auf die Positionen des prospektiven Koalitionspartners zubewegen? Oder treten sie - unbeeinflusst von Koalitionspräferenzen - mit einem eigenständigen Programm an, das erst in den späteren Koalitionsverhandlungen mit den Positionen des Partners abgeglichen wird? Thomas Bräuninger und Marc Debus ${ }^{40}$ weisen zu Recht darauf hin, dass es nicht sinnvoll wäre, von einem eigenständigen Einfluss der politischen Inhalte auf die Koalitionsbildung auszugehen, wenn die erstgenannte Hypothese zuträfe. Tatsächlich können die Parteien jedoch dem Dilemma nicht entrinnen, das ihnen durch die Koalitionssignale unter Wettbewerbsgesichtspunkten entsteht. Auf der einen Seite müssen sie auf eine Erwiderung der Signale hoffen, da nur auf dieser Basis eine Koalition überhaupt gebildet werden kann. Dafür könnten unter Umständen programmatische Zugeständnisse erforderlich sein. Auf der anderen Seite laufen sie Gefahr, sich bei einer zu starken Annäherung an den Partner der eigenen Anhängerschaft zu entfremden. Auch mit Blick auf das Ziel einer gemeinsamen Mehrheit wäre eine zu große programmatische Nähe womöglich kontraproduktiv. Mehrere Parteien, die innerhalb eines bestimmten Spektrums auftreten und im Wettbewerb unterschiedliche personelle und programmatische Angebote machen, können ein gegebenes Wählerpotenzial umfassender ausschöpfen als eine Partei allein. Deshalb empfiehlt es sich, die programmatische Anpassung nicht zu weit zu treiben. Hätte die Union diese Lektion 2005 durch einen etwas weniger neoliberal akzentuierten Bundestagswahlkampf beherzigt, wäre ihre Wunschkoalition mit der FDP wahrscheinlich zustande gekommen.

Aus dem Beispiel lassen sich allerdings weder im bundesdeutschen Kontext noch im Kontext anderer vergleichbarer Koalitionsdemokratien irgendwelche allgemeinen Schlüsse

39 Vgl. Franz Urban Pappi / Alexander Herzog / Ralf Schmitt, a.a.O. (Fn. 16), S. 509 f.

40 Vgl. Thomas Bräuninger / Marc Debus, Der Einfluss von Koalitionsaussagen, programmatischen Standpunkten und der Bundespolitik auf die Regierungsbildung in den deutschen Ländern, in: PVS, 49. Jg. (2008), H. 2, S. $309-338$, S. 324. 
ziehen. Dass Debus' ${ }^{\prime 1}$ Versuch, die These einer vorauseilenden programmatischen Anpassung an die gewünschten Koalitionspartner empirisch zu belegen, zu keinen eindeutigen Ergebnissen führen würde, war absehbar. Einerseits sind die Akteurskonstellationen viel zu unterschiedlich - jeder Fall liegt anders und kann deshalb nur einzeln betrachtet werden. Andererseits weiß man nicht, ob die programmatischen Anpassungen tatsächlich koalitionspolitisch motiviert sind oder ob sie eher mit Blick auf den Wähler vorgenommen werden (um die eigenen Stimmen zu maximieren). Methodisch stellen sich hier dieselben Probleme wie bei der Analyse der elektoralen Wirkungen der Koalitionssignale.

Das ändert aber nichts an der Bedeutung des eigentlichen Sachverhalts. Die künftigen Koalitionsparteien stehen im Wettbewerb vor einer schwierigen Gratwanderung. Sie müssen mit dem heutigen Gegner und morgigen Partner möglichst pfleglich umgehen und sich gleichzeitig durch die Arbeit am eigenen Profil von diesem abgrenzen. Zum pfleglichen Umgang gehört nicht nur, dass man auf persönliche Angriffe und übertriebene Polemik verzichtet, sondern auch, dass keine unnötigen inhaltlichen Stolpersteine aufgebaut werden, die in den anschließenden Koalitionsverhandlungen einer Einigung im Wege stehen könnten. ${ }^{42}$ Die eigene Profilierung wiederum darf nicht so weit gehen, dass die ursprünglichen Positionen in den am Ende gefundenen Kompromissen bis zur Unkenntlichkeit entstellt werden. Was sie vorher an Kredit bei der eigenen Wählerschaft gewonnen oder behalten hätte, würde eine Partei dann nachher umso rascher verspielen. Dass Koalitionskompromisse häufig als Feigenblatt herhalten müssen, um unhaltbare Versprechungen später als lässliche Wahlkampfsünde abzutun, hat der SPD-Vorsitzende Franz Müntefering kurz nach Regierungsantritt der Großen Koalition mit entwaffnender Ehrlichkeit demonstriert, als er forderte, die Politiker dürften doch bitte nicht an ihren Wahlkampfaussagen gemessen werden. Offenbar sind auch die Konsensdemokratien gegen die Bedrohungen eines populistischen Überbietungswettlaufs nur begrenzt gefeit.

\section{Koalitionssignale vor der Bundestagswahl 2009: ein Ausblick}

Koalitionsaussagen werden zu einem Problem, wenn sie die Koalitionsbildung nicht erleichtern, sondern erschweren. Die positiven Signale sind deshalb unter normativen Gesichtspunkten den negativen vorzuziehen: Die Parteien müssen sich so zueinander positionieren, dass am Ende mindestens eine gangbare Koalitionsoption übrig bleibt. Je breiter die positiven Koalitionssignale im Vergleich zu den tatsächlich geschlossenen Koalitionen gestreut werden, desto größer ist die Gewähr, dass eine Koalition am Ende zustande kommt. Bei Vorhandensein mehrerer Alternativen wird die Koalitionsbildung nicht zwangsläufig leichter, im Gegenteil: Besteht unter den Optionen keine klare Hierarchie und / oder blockieren sie sich gegenseitig, kann der Prozess unter Umständen sehr langwierig werden, wie

41 Vgl. Marc Debus, a.a.O. (Fn. 30), S. $194 \mathrm{ff}$.

42 Dass im Rahmen einer solchen Forderung zumindest im bundesdeutschen Kontext immer noch vieles möglich ist, zeigt das Beispiel Kernenergie. Auch wenn die Union die Gelegenheit nutzte, das Thema im Wahlkampf aufzugreifen, wäre das für sie wahrscheinlich kein Grund, ein Bündnis mit der SPD oder selbst den Grünen zu verweigern. Gegen deren Willen könnte sie eine Korrektur des Ausstiegsbeschlusses ohnehin nicht durchsetzen. Das Thema bleibt also rein symbolisch. In dieser Eigenschaft erfüllt es aber für beide Seiten eine nützliche Funktion, indem es zur Mobilisierung der jeweiligen Wählerklientel beiträgt. 
das belgische Beispiel gerade wieder gezeigt hat. Aus normativer Sicht empfiehlt sich hier eine Abstufung der Aussagen, die einerseits die eigenen Prioritäten deutlich macht und andererseits die verschiedenen Szenarien des Wahlausgangs berücksichtigt.

In der Bundesrepublik bereitete die Regierungsbildung auf beiden Ebenen des politischen Systems lange Zeit keine Schwierigkeiten. Sowohl im Dreiparteiensystem der sechziger und siebziger als auch im Vierparteiensystem der achtziger Jahre sorgte die Verbindung von klaren Koalitionssignalen mit der Arithmetik der Wahlergebnisse für einen Automatismus der Mehrheitsbildung. Lediglich die kurze Phase von 1980 bis 1985 muss hiervon zum Teil ausgenommen werden. Weil die SPD Koalitionen mit den Grünen in dieser Zeit noch nicht als machbar erachtete, konnten Situationen eintreten, in denen die für eine kleine Zweier-Koalition notwendige Mehrheit nicht mehr vorhanden war. Dies war zum Beispiel 1982 und 1986 in Hamburg der Fall.

Das Hinzutreten der Grünen führte das Parteiensystem auf eine noch stärker bipolare Struktur zurück, als sie bis dahin gegeben war. Die ihrer Scharnierfunktion beraubte FDP wurde nun koalitionspolitisch einseitig an die Union gebunden, während sich die Grünen der SPD als einzigem potenziellen Koalitionspartner verschrieben. ${ }^{43}$ Abweichungen von diesem Muster waren selten. Eine Folge der Bipolarisierung war, dass 1998 zum ersten Mal auf Bundesebene ein ausschließlich von den Wählern herbeigeführter vollständiger Regierungswechsel zustande kam.

Das Überleben der postkommunistischen PDS als ostdeutsche Regionalpartei führte nach der deutschen Einheit zu einer Viereinhalb-Parteienstruktur. Die Parameter der Koalitionsbildung im Bund wurden dadurch zunächst nicht verändert, da die PDS auf gesamtdeutscher Ebene noch zu schwach blieb, um die Bildung der vertrauten kleinen ZweierKoalitionen nach den Bundestagswahlen zu vereiteln. Auf der Landesebene entwickelten sich die Parteiensysteme und Koalitionsmodelle dagegen auseinander. Im Westen, wo die PDS in elektoraler Bedeutungslosigkeit verharrte, blieb es bei der bipolaren Struktur der achtziger Jahre, während im Osten ab Mitte der neunziger Jahre eine gleichgewichtige Dreiparteienstruktur entstand, in der die PDS zu Union und SPD allmählich aufschließen konnte. Damit ließ sich auch die Frage einer Regierungsbeteiligung der PDS nicht mehr umgehen.

Die eigentliche Zäsur der Parteiensystementwicklung auf Bundesebene markiert die Entstehung der gesamtdeutschen Linkspartei im Jahre 2005. ${ }^{44}$ Weil die nach Westen erweiterte PDS nun bundesweit auf Stimmenanteile in einer Größenordnung von zehn Prozent hoffen kann (was einer Verdoppelung ihres vormaligen Wähleranteils entspricht), sind Mehrheiten für eine kleine Zweier-Koalition sehr viel unwahrscheinlicher geworden. Damit unterscheidet sich die Situation im Bund sowohl von derjenigen der alten Bundesländer hier bleibt bei einer schwächeren Linkspartei die Bildung von Zweier-Koalitionen tendenziell immer noch möglich -, als auch von derjenigen Ostdeutschlands, wo neben der Großen Koalition auch Bündnisse aus SPD und Linken denkbar sind. Das Auseinanderfallen von Bundes- und Landesebene ist deshalb prekär, weil die Länderpolitik damit als Testlabor für neue Koalitionen auszufallen droht. Wenn lagerübergreifende Dreier-Koalitionen von

43 Vgl. Uwe Jun, Parteiensystem und Koalitionskonstellationen vor und nach der Bundestagswahl 2005, in: Frank Brettschneider / Oskar Niedermayer / Bernhard Weßels (Hrsg.), Die Bundestagswahl 2005, Wiesbaden 2007, S. 491 - 515, S. 497.

44 Vgl. Frank Decker, Die Zäsur, in: Berliner Republik, 7. Jg. (2005), H. 6, S. 66 - 71. 
SPD, FDP und Grünen oder Union, FDP und Grünen ${ }^{45}$ eine Alternative zur jetzigen Großen Koalition bereithalten sollen, müssten sie von den Parteien also ohne vorherigen Probelauf in den Ländern in Angriff genommen werden.

Obwohl über die Dreier-Koalitionen schon am Wahlabend des 18. September 2005 munter spekuliert wurde, standen sie damals nicht wirklich in Reichweite - die Debatte darüber hatte insofern eine eher „heuristische“ Funktion. Aus Sicht der FDP hätten beide Optionen den zuvor ausgesendeten Koalitionssignalen widersprochen, die Rot-Grün eine klare Abfuhr erteilt hatten. So als ob sie ihr Stigma der „Umfaller-Partei“, das ihnen seit 1961 anhaftete, für alle Zeiten abschütteln wollten, wurden die Liberalen deshalb nicht müde, sich vor der Wählerschaft als Partei hinzustellen, die „ihr Wort hält“.

Die Erwartung, dass die schwierige Regierungsbildung zu einer Flexibilisierung der Koalitionsbereitschaft führen würde, ist bei den seitherigen Landtagswahlen nur zum Teil erfüllt worden. ${ }^{46}$ In Hamburg kam es im Frühjahr 2008 zur ersten schwarz-grünen Koalition auf Landesebene, wodurch das Tor für ein Jamaika-Bündnis ein Stück geöffnet werden konnte. Bei der kurz vorher stattgefundenen Wahl in Hessen hatten sich die Parteien mit negativen Koalitionsaussagen dagegen noch überboten. Dies ging soweit, dass selbst eine Große Koalition von der Union wie der SPD als Möglichkeit ausgeschlossen wurde. So wie die Große Koalition hätten auch sämtliche Varianten eines Dreier-Bündnisses den Koalitionssignalen widersprochen. Dass Andrea Ypsilanti in diesem Mikado-Spiel als erste den Wortbruch riskierte, sollte ihr und der Landes-SPD zum Verhängnis werden.

Am Machtpoker in Hessen lassen sich viele der zuvor hypothesenartig dargestellten $\mathrm{Zu}$ sammenhänge exemplarisch nachzeichnen. Um einen Ausweg aus der verfahrenen Situation zu finden, glaubte die SPD, sich über ihre negativen Koalitionssignale hinwegsetzen zu müssen. Dass die deutsche Öffentlichkeit dies als offenen Wortbruch nahezu einhellig verurteilte, verweist auf die hohe Bedeutung, die den Koalitionsaussagen hierzulande beigemessen wird. Hätten die Sozialdemokraten deshalb besser daran getan, auf eine negative Koalitionsaussage zu verzichten? Dies zu bejahen setzt voraus, dass ihr ein Offenhalten der Koalitionsfrage an der Wahlurne nicht geschadet hätte. Angesichts der innerparteilichen Zerrissenheit der SPD im Umgang mit der Linken scheint das eine ziemlich verwegene Annahme. Ein Koalitionssignal, das von einer starken Minderheit der Partei und einem erheblichen Teil ihrer Wählerschaft nicht mitgetragen wird, hätte von vornherein nur ein schwaches sein können.

Nachdem das Tabu gebrochen und die koalitionspolitische Öffnung gegenüber der Linken auch in den alten Ländern erfolgt ist, muss die SPD die sich daraus ergebenden unangenehmen Konsequenzen für den Bundestagswahlkampf ertragen. Gewiss mag es gute inhaltliche Gründe geben, eine Zusammenarbeit zwischen SPD und Linken im Bund anders zu betrachten als auf Landesebene, in den ostdeutschen Ländern anders als in den westdeutschen und bei der Wahl des Bundespräsidenten anders als bei der Wahl des Kanzlers. Ob

45 Weil CDU und CSU organisatorisch selbständige Parteien sind, handelt es sich bei der JamaikaKoalition genau genommen um ein Vierer-Bündnis und bei der jetzigen Großen Koalition um ein Bündnis aus drei Partnern. Abgelesen werden kann dies u.a. daran, dass die bayerische Schwester im Koalitionsausschuss gleichberechtigt vertreten ist und in allen unionsinternen Entscheidungen ein Vetorecht besitzt.

46 Vgl. Frank Decker, Ankunft im Vielparteienstaat, in: Berliner Republik, 10. Jg. (2008), H. 2, S. $19-25$. 
sich diese Gründe der Wählerschaft tatsächlich vermitteln lassen, ist aber fraglich. Die SPD wird also mit einer gewaltigen Gegenmobilisierung in der Koalitionsfrage rechnen müssen. Dies gilt umso mehr, als die Landtagswahlen im Saarland und in Thüringen, wo es zu rotroten Koalitionen kommen könnte, mitten in den Bundestagswahlkampf hineinfallen.

Vom Funktionieren der Linksbündnisse und programmatischen Lernprozess der Linken wird es abhängen, ob die Bundesrepublik nach 2009 in eine neue Ära der Bipolarisierung ihres Parteiensystems eintritt, oder ob eine multiple Koalitionslandschaft mit lagerübergreifenden Bündnissen das Bild prägt. Wie schwierig es einstweilen noch ist, letztere zustande zu bringen, haben die Landtagswahlen in Hessen gezeigt. Durchaus als Ironie der heutigen Parteiensystemstruktur darf gelten, dass FDP und Grüne die Funktion eines Scharniers, das den Wechsel zwischen Union und SPD ermöglicht, nun gemeinsam ausüben. Das Problem besteht nur darin, dass die beiden Teile des Scharniers in verschiedene Richtungen streben: Die Liberalen möchten nicht gerne Mehrheitsbeschaffer von Rot-Grün und die Grünen nicht das Anhängsel einer bürgerlichen Koalition sein.

Vergleicht man die bisher mit Blick auf die Bundestagswahl 2009 gesendeten Koalitionssignale mit jenen vor der Wahl 2005, lässt sich durchaus eine größere Offenheit der Beteiligten für neue Optionen feststellen. Union und SPD bleiben die jeweiligen Wunschpartner von FDP und Grünen, die eine Zusammenarbeit mit der ihnen ferner stehenden Volkspartei inzwischen aber ebenfalls für denkbar halten. Nimmt man die auf den Parteitagen von Grünen und FDP (im Mai 2009) getroffenen Beschlüsse als Gradmesser, gilt das allerdings nicht im selben Maße. Während die Parteispitze der Grünen unter dem Druck ihrer Basis gehalten war, einem Jamaika-Bündnis eine Absage zu erteilen, scheint sich die FDP die Hintertür einer Ampelkoalition - anders als 2005 - vor der Wahl bewusst offenzuhalten. Ob sie das Koalitionssignal der SPD, die ihre Präferenz für die Ampel ausdrücklich erklärt hat, nach der Wahl tatsächlich erwidert und damit den Weg für die Ablösung der Großen Koalition bereitet, weiß wahrscheinlich noch nicht einmal ihr Vorsitzender Westerwelle selbst, von dessen eigenen Ambitionen die Koalitionsentscheidung maßgeblich abhängen dürfte. Auf einem anderen Blatt steht die Frage, ob ein Dreierbündnis von SPD, FDP und Grünen angesichts der Mehrheitsverhältnisse im Bundesrat überhaupt regierungsfähig wäre, wo es derzeit (Juni 2009) über ganze sieben Stimmen verfügt. Es ist kaum anzunehmen, dass eine aus der Regierung herauskatapultierte Union eine besondere Neigung verspüren würde, mit der neuen Mehrheit zusammenzuarbeiten. Stellt man diese

Tabelle 2: Koalitionssignalmatrix für die Bundestagswabl 2009

\begin{tabular}{|l|c|c|c|c|c|}
\hline & CDU/CSU & SPD & FDP & Grüne & Linke \\
\hline CDU/CSU & - & 0 & +2 & $0^{*}$ & -2 \\
\hline SPD & 0 & - & $+1^{*}$ & +2 & $-2^{*}$ \\
\hline FDP & +2 & $0^{*}$ & - & 0 & -2 \\
\hline Grüne & $-2^{*}$ & $+2^{*}$ & 0 & - & $-1^{*}$ \\
\hline Linke & -2 & $+1^{*}$ & -2 & $+1^{*}$ & - \\
\hline
\end{tabular}

Anmerkung: Die „Zeilenpartei“ macht eine Aussage zur „Spaltenpartei“. 0 steht für kein erkennbares, 1 für ein implizites und 2 für ein ausdrückliches Koalitionssignal. Das Vorzeichen macht deutlich, ob es sich um ein positives oder negatives Koalitionssignal handelt. Die asymmetrischen Koalitionssignale sind mit Sternchen versehen.

Quelle: Darstellung in Anlehnung an Eric Linhart, a.a.O. (Fn. 4), S. 472 f. 
Widrigkeiten mit in Rechnung, wäre es unter Regierungsgesichtspunkten (nicht unbedingt unter Demokratiegesichtspunkten) vermutlich besser, die SPD würde als Juniorpartner in der Großen Koalition verbleiben. Die Union dürfte ohnehin kein Interesse haben, die Unwägbarkeiten eines Jamaika-Bündnisses gegen die relative Verlässlichkeit der bestehenden Regierung einzutauschen, in der sie ja als wahrscheinlich stärkerer Partner weiter die Kanzlerin stellen könnte. Unterstellt, dass es für eine bürgerliche Koalition von Union und FDP auch dieses Mal keine Mehrheit gibt, verbleiben für die Koalitionsbildung im September mithin nur zwei realistische Alternativen: ein Ampelbündnis oder die Große Koalition.

\section{Institutionelle Konsequenzen}

Ob ein gegnerschaftliches oder konsensuelles System des Parteienwettbewerbs vorzuziehen sei, ist unter deutschen Politologen umstritten. Die Anhänger des erstgenannten mehrheitsdemokratischen Modells können der Erweiterung des Parteiensystems um die gesamtdeutsche Linke durchaus etwas Positives abgewinnen, denn sie birgt die Perspektive einer klaren bipolaren Struktur: Die bürgerlichen Parteien Union und FDP würden danach den drei linken Parteien als koalitionspolitisch zusammengebundene, streng voneinander abgegrenzte Lager gegenüberstehen. Andere Verfechter der Mehrheitsdemokratie suchen das Heil demgegenüber eher in einer Wahlrechtsreform nach britischem oder französischem Vorbild. ${ }^{47}$ Weil eine solche Reform den erbitterten Widerstand der kleinen Parteien nach sich ziehen und auch in anderer Hinsicht mit den institutionellen Strukturen des „Parteienbundesstaates" kollidieren würde, erscheint sie wenig realistisch. Als manipulativer Eingriff in die bestehenden Regelungen wäre sie legitimatorisch zudem kaum begründbar, denn es würden gerade die Verlierer des Parteiensystemwandels - die beiden Noch-Volksparteien von ihr profitieren. Die Idee eines künstlich herbeigeführten mehrheitsdemokratischen Schnitts kann daher an dieser Stelle beiseite gelassen werden. ${ }^{48}$

Die Gegenposition sieht in den durch die Pluralisierung des Parteiensystems verursachten Konsenszwängen nichts Negatives. Sie betrachtet diese im Gegenteil als Chance für einen stärker sachorientierten Politikstil. Die Bewertung fällt dabei allerdings für die verschiedenen Modelle der Konsensbildung sehr unterschiedlich aus. Am besten schneiden die lagerübergreifenden Dreierkoalitionen ab, denen zwar eine größere Instabilität zugeschrieben wird als den bisherigen Zweierbündnissen, die sich aber ansonsten gut in die Funktionslogik des parlamentarischen Regierungssystems einfügen. Am schlechtesten wird die Große Koalition bewertet, bei der genau dies nicht der Fall ist. Große Koalitionen fördern zwar den Konsensualismus, doch tun sie dies, indem sie die Gewichte zwischen Regierungsmehrheit und Opposition zu Lasten der letzteren einseitig verschieben. Im Bundestag führt das zum Beispiel gegenwärtig dazu, dass den Oppositionsparteien ein wesentlicher Teil der parlamentarischen Kontrollrechte - etwa die Einleitung eines abstrakten Normen-

47 Vgl. Gerd Strohmeier, Ein Plädoyer für die „gemäßigte Mehrheitswahl“: optimale Lösung für Deutschland, Vorbild für Österreich und andere Demokratien, in: ZParl, 38. Jg. (2007), H. 3, S. $578-590$.

48 Vgl. Frank Decker, Konsens- oder mehrheitsdemokratischer Wandel des Parlamentarismus? Eine Replik auf Gerd Strohmeier in Heft 3/2007 der ZParl, in: ZParl, 38. Jg. (2007), H. 4, S. 857 $-861$. 
kontrollverfahrens ${ }^{49}$ oder die Einsetzung eines Untersuchungsausschusses - vorenthalten bleiben, die von mindestens einem Drittel beziehungsweise einem Viertel der Abgeordneten unterstützt werden müssen. Darüber hinaus wird das Prinzip der alternierenden Regierung, auf dem die parlamentarischen und präsidentiellen Systeme gleichermaßen basieren, durch Große Koalitionen faktisch außer Kraft gesetzt, die allenfalls einen „halben“ Regierungswechsel gestatten. Die Folge ist eine „Störung“ des normalen Sanktionswahleffekts und das sprichwörtliche Erstarken der Ränder. ${ }^{50}$ Große Koalitionen finden deshalb in der Regel nur als Ausnahmefall Akzeptanz, der spätestens nach einer Wahlperiode wieder zu beenden sei. Werden sie als Regierungsformat perpetuiert, laufen sie Gefahr, die Legitimationsgrundlagen des demokratischen Systems auszuhöhlen. ${ }^{51}$

Ein flexibleres Format als die Großen Koalitionen hält der aus den skandinavischen Ländern geläufige Minderheitsparlamentarismus bereit. ${ }^{52}$ Minderheitsregierungen sind dadurch charakterisiert, dass exekutive und legislative Koalitionen auseinanderfallen. Die Regierung wird von einer Mehrheit des Parlaments bestellt und im Amt gehalten (indem man auf die Einbringung oder Unterstützung eines Misstrauensvotums verzichtet), doch treten nicht alle Teile des Parlaments, die die Regierung tragen, ihr auch formell bei. ${ }^{53}$ Wichtig ist die Unterscheidung zwischen gestützten und „lediglich“ geduldeten Minderheitsregierungen, was gerade in Deutschland oft verwechselt wird. ${ }^{54}$ Bei ersteren verabreden die Regierungspartei oder die Regierungsparteien mit dem stützenden Partner eine feste Zusammenarbeit in Gestalt eines Gesetzgebungsprogramms, die in Form und Wirkung einer Koalition ähnelt. Bei einer lediglich geduldeten Minderheitsregierung erfolgt die Zusammenarbeit

49 Hier mag einer der Gründe dafür liegen, warum das formelle und materielle Prüfungsrecht des Bundespräsidenten, das mit Blick auf die Normenkontrollfunktion des Verfassungsgerichts normalerweise nicht bemüht werden muss, unter den Bedingungen einer Großen Koalition stärker ins Spiel kommt. Tatsächlich wurden in der zu Ende gehenden Wahlperiode zwei Gesetze von Amtsinhaber Horst Köhler zum Unwillen der Regierung nicht ausgefertigt.

50 Melanie Haas, Auswirkungen der Großen Koalition auf das Parteiensystem, in: APuZ, B 35 / 36 (2007), S. $18-26$.

51 Vgl. Wilhelm Hennis, Große Koalition ohne Ende? Die Zukunft des parlamentarischen Regierungssystems und die Hinauszögerung der Wahlrechtsreform, München 1968. Am österreichischen Beispiel lassen sich diese Probleme gut illustrieren. Obwohl sie das Prinzip der Alternanz bis zum Rande strapazierten, wurden die Großen Koalitionen als Ausdruck der Proporz- oder Konkordanzdemokratie in der Alpenrepublik lange Zeit gutgeheißen. In dem Maße, wie die gesellschaftlichen Grundlagen der Lagerstruktur erodierten, geriet das Regierungsformat unter Druck. Dass es in den 1990er Jahren dennoch aufrechterhalten werden musste, war eine paradoxe Folge des Aufstiegs der konkordanzfeindlichen Haider-FPÖ. Vgl. Hubertus Czernin, Der Haider-Macher. Franz Vranitzky und das Ende der alten Republik, Wien 1997. Die Destabilisierung der Regierungsverhältnisse lässt sich auch an der Häufung vorgezogener Neuwahlen ablesen (seit 1990 jede zweite). Betrachtet man alle Regierungen der Zweiten Republik, waren die Großen Koalitionen kurzlebiger als die übrigen Regierungsformate, obwohl sie im Ganzen länger amtierten (36 gegenüber 28 Jahre).

52 Vgl. Wolfgang Renzsch / Stefan Schieren, Große Koalition oder Minderheitsregierung: SachsenAnhalt als Zukunftsmodell des parlamentarischen Regierungssystems in den neuen Bundesländern, in: ZParl, 28. Jg. (1997), H. 3, S. $391-407$.

53 Vgl. Winfried Steffani, Regierungsmehrheit und Opposition, in: ders. (Hrsg.), Regierungsmehrheit und Opposition in den Staaten der EG, Opladen 1991, S. 11 - 35, S. 24 ff.

54 Vgl. Eberhard Schütt-Wetschky, Verhältniswahl und Minderheitsregierungen. Unter besonderer Berücksichtigung Großbritanniens, Dänemarks und der Bundesrepublik Deutschland, in: ZParl, 18. Jg. (1987), H. 1, S. $94-109$. 
dagegen auf einer ad hoc-Basis. Hier kann sich die Regierung auf die Unterstützung des duldenden Teils nicht immer sicher verlassen, der dafür aber im Gegenzug bereit ist zu akzeptieren, dass sich die Regierung die Unterstützung gegebenenfalls auch bei anderen Fraktionen besorgt. Ein solches Tolerierungsmodell erlaubt und ermöglicht mithin ein Regieren mit wechselnden legislativen Mehrheiten. Insofern weist es Ähnlichkeiten mit der Funktionsweise des präsidentiellen Systems auf. ${ }^{55}$

In Deutschland wird Minderheitsregierungen in der Regel eine hohe Krisenanfälligkeit zugeschrieben, weil man sie fälschlicherweise mit dem Duldungsmodell gleichsetzt. Tatsächlich ist diese Variante in Europa jedoch - abgesehen von Dänemark - kaum verbreitet. Die meisten Minderheitsregierungen unterscheiden sich in der Praxis also nicht sonderlich von den normalen Koalitionsregierungen und stehen diesen auch in ihren Stabilitätseigenschaften nicht nach. ${ }^{56}$

Der Mehrheitsdogmatismus in der parlamentarischen Kultur der Bundesrepublik hat dazu geführt, dass Minderheitsregierungen hierzulande allenfalls als kurzzeitige Übergangsregierungen amtierten (deren Abberufung sich nicht lohnte, weil die Bildung einer neuen Mehrheitsregierung bereits im Gange war). Als reguläres Regierungsformat wurden und werden sie demgegenüber auch von vielen Vertretern der Politikwissenschaft weiter mit Argwohn betrachtet, wie die Auseinandersetzung um das „Magdeburger Modell“ einer von der PDS geduldeten Minderheitsregierung in Sachsen-Anhalt oder die Diskussion um die Rolle des Südschleswigschen Wählerverbandes (SSW) bei der Regierungsbildung in Schleswig-Holstein gezeigt haben. ${ }^{57}$ Beide Fälle werfen zugleich ein bezeichnendes Licht auf die institutionelle Gleichförmigkeit der Landes- und Bundespolitik, die einer der Gründe dafür ist, warum die Koalitionsbildungen in den Ländern permanent durch die Brille des Bundes betrachtet werden. ${ }^{58}$ Diese Gleichförmigkeit ist durch die jeweiligen Eigenarten der Bundes- und Landespolitik keinesfalls gedeckt. Die Hauptaufgaben der Länder liegen im deutschen Verbundföderalismus bekanntlich im Verwaltungsbereich. Ein konsensueller Politikstil ist dadurch eher möglich als in der Bundespolitik, wo die Gesetzgebung im Mittelpunkt steht. Das dualistische Regierungsmodell des parlamentarischen Systems, das auf der Bundesebene seinen Sinn haben mag, geht insofern an der Regierungswirklichkeit der Länder vorbei.

Doch wie lauten die Alternativen? Der radikalste Vorschlag sieht vor, das parlamentarische System in den Ländern abzuschaffen und durch ein präsidentielles System zu ersetzen. Die Ministerpräsidenten würden danach getrennt von den Landtagen direkt gewählt. Die-

55 Vgl. Nicole Bolleyer, Minderheitsparlamentarismus - eine akteurszentrierte Erweiterung der Parlamentarismus-Präsidentialismustypologie, in: ZPol, 11. Jg. (2001), H. 4, S. $1519-1546$.

56 Vgl. Kaare Strøm, Majority Rule and Minority Government, Cambridge 1990.

57 Vgl. Werner J. Patzelt / Roland Schirmer, Parlamentarismusgründung in den neuen Bundesländern, in: APuZ, B 27 (1996), S. 20 - 28; Everhard Holtmann, Dürfen die das, wo sie doch Dänen sind? Über den Umgang mit Macht und Minderheiten in Deutschland, in: ZParl, 36. Jg. (2005), H. 3, S. $616-629$.

58 Altkanzler Helmut Schmidt hat sich in seiner Kolumne im Zeit-Magazin darüber zu Recht mokiert. „Die sechzehn Bundesländer brauchen nicht notwendig Regierung und Opposition; vielmehr ist ihnen eine anständige Verwaltung notwendig und ebenso ein Landtag, der die Verwaltung sorgfältig überwacht. Das Problem der Koalitionsbildung stellt sich dagegen im Bund, denn in Berlin muss wirklich regiert werden." Ders., Auf eine Zigarette mit Helmut Schmidt. Über Koalitionen und italienische Zustände, in: Zeit-Magazin vom 21. Februar 2008, S. 54. 
ses Modell orientiert sich an der Regierungsform der - von ihrem Aufgabenzuschnitt mit den Ländern vergleichbaren - Kommunen, wo die Direktwahl der Bürgermeister in den neunziger Jahren flächendeckend eingeführt worden ist. ${ }^{59}$ Der weniger radikale Vorschlag geht dahin, das bestehende parlamentarische Modell zu flexibilisieren. Dies gilt vor allem in Bezug auf die Minderheitsregierungen, die auf der Landesebene positiver zu bewerten sind als im Bund und deshalb als Regierungsformat offensiv angestrebt werden sollten. Vor diesem Hintergrund ist es paradox und aus heutiger Sicht kaum nachvollziehbar, dass im Zuge von Verfassungsgebungs- und -reformprozessen zwölf der 16 Bundesländer die Zulassung von Minderheitsregierungen durch die Einführung so genannter Oppositionsklauseln sogar erschwert haben. ${ }^{60}$

Auch die Etablierung bestimmter Regeln bei der Regierungsbestellung würde die Parteien von der Notwendigkeit, Koalitionsaussagen treffen zu müssen, zum Teil entlasten. Der Automatismus der Mehrheitsbildung hat dafür gesorgt, dass es bislang solcher Regeln im Normalfall nicht bedurfte. Weder gab und gibt es in Deutschland die Institution eines Regierungsformateurs, noch musste man auf das ungeschriebene Gesetz zurückgreifen, wonach der Regierungsauftrag im parlamentarischen System stets der stärksten Partei beziehungsweise Fraktion gebührt. In einem System multipler Koalitionen wird man nicht umhinkommen, entsprechende Vorkehrungen zu treffen. Dabei kann man entweder auf die Erfahrungen anderer Länder bauen, die mit Mehrparteienkoalitionen vertraut sind (Benelux, Skandinavien). Oder man verständigt sich auf Regeln, die den besonderen Bedingungen des deutschen Parteiensystems Rechnung tragen. ${ }^{61}$

Noch grundlegender sind die Rückwirkungen, die sich aus der Pluralisierung der Koalitionslandschaft mit Blick auf die Regierungsfähigkeit ergeben. Sie betreffen die Zustimmungsbedürftigkeit eines Großteils der Gesetzgebung im Bundesrat. Hier führt die Häufung der "gemischten“ oder „neutralen“ Koalitionen in den Ländern dazu, dass sich das Blockadeproblem tendenziell verschärft, weil die in den Koalitionsverträgen vorgesehenen Enthaltungen (im Falle der Nichteinigung auf das Abstimmungsverhalten) einer Ablehnung der Gesetzesvorlage gleichkommen. Um den Weg in eine faktische Allparteienregierung zu vermeiden, bieten sich als Antwort entweder eine Änderung der Abstimmungsre-

59 Vgl. Frank Decker, Direktwahl der Ministerpräsidenten?, in: Recht und Politik, 37. Jg. (2001), H. 3, S. $152-161$.

60 Vgl. Sabine Kropp, Oppositionsprinzip und Mehrheitsregel in den Landesverfassungen. Eine Analyse am Beispiel des Verfassungskonflikts in Sachsen-Anhalt, in: ZParl, 28. Jg. (1997), H. 3, S. 373 - 390; Sven Thomas, Zur Handlungsfähigkeit von Minderheitsregierungen am Beispiel des „Magdeburger Modells“, in: ZParl, 34. Jg. (2003), H. 4, S. 792 - 806. Als Argument für das Festhalten an der parlamentarischen Regierungsform wird von Länderseite häufig die Beteiligung an der Bundesgesetzgebung ins Feld geführt. Tatsächlich würde die Einführung der Direktwahl unweigerlich Konsequenzen für die Struktur der Zweiten Kammer nach sich ziehen, die in ihrer heutigen rein exekutivischen Form so nicht bestehen bleiben könnte. Die Minderheitsregierungen würden das strukturelle Problem demgegenüber gerade entschärfen, weil es dem stützenden oder duldenden Partner nicht zusteht, das Land im Bundesrat zu vertreten. Dies könnte auch die Glaubwürdigkeit einer differenzierten Koalitionsstrategie (im Bund nein, in den Ländern ja) befördern, die sich den Wählern - wie die SPD zurzeit leidvoll erfährt - ansonsten nur schwer vermitteln lässt.

61 Stehen Schwarz-Gelb und Rot-Grün als gewünschte Koalitionen gegeneinander, könnte man den Regierungsauftrag zum Beispiel der stärkeren Formation zusprechen (statt der stärksten Partei). 
geln im Bundesrat an ${ }^{62}$ oder eine Abkehr vom bisherigen Enthaltungsautomatismus in den gemischt regierten Ländern.

Nicht minder prekär sind die Folgen des neuen Parteiensystems unter Demokratiegesichtspunkten. Institutionelle Regeln und die öffentlich erzeugte Erwartung, sich koalitionspolitisch zu bekennen, sind zwar aus demokratischer Sicht zu begrüßen; sie ändern aber nichts daran, dass in einem System multipler Koalitionen der Einfluss des Wählers auf die Regierungsbildung zurückgeht. Die Oligarchisierung der Parteiendemokratie könnte deshalb den Druck erhöhen, die Legitimationsdefizite an anderer Stelle auszugleichen. Dazu kämen zum einen die Einführung beziehungsweise der Ausbau von plebiszitären Beteiligungsformen auf der staatlichen Ebene in Betracht, die in der Bundesrepublik bislang ein Schattendasein fristen, zum anderen die Stärkung der Mitgliederrechte in den Parteien selbst und die Abkehr vom heutigen reinen Delegiertensystem.

\section{Perspektiven der Forschung}

Dass die Politikwissenschaft bei der Erforschung der hier erörterten Fragen noch in den Kinderschuhen steckt, hat zum einen Teil mit methodologischen Problemen zu tun. So wie die Koalitionssignale nur einer von mehreren Faktoren sind, die die Koalitionsbildung, das Wählerverhalten und die programmatisch-strategische Positionierung der Parteien bestimmen, so sind sie selbst ebenfalls einer Reihe unterschiedlicher Einflussfaktoren unterworfen. Quantitativ-statistische Verfahren bieten sich an, um die erwarteten Zusammenhänge empirisch zu messen (beziehungsweise zu schätzen), führen allerdings nicht immer zum Ziel. Gerade bei der Bestimmung der institutionellen und politisch-kulturellen Einflüsse sollten die quantitativen Vergleiche (mit möglichst vielen Fällen) deshalb um qualitative Untersuchungen ergänzt werden, die mit wenigen ausgesuchten Fällen auskommen.

Das andere Problem betrifft die Daten. Nachdem sich die Koalitionssignale - wie oben gezeigt - relativ leicht operationalisieren lassen, können diese für die aktuellen und künftigen Wahlen ohne größere Schwierigkeiten ermittelt werden. Für die vergangenen Wahlen stehen entsprechende Daten aber aufgrund des Desinteresses der Forschung an den Koalitionsaussagen nur rudimentär zur Verfügung. Die nachträgliche Rekonstruktion der Signale dürfte sich vor allem bei den weit zurückliegenden Wahlen sehr mühsam gestalten und einen Einzelforscher rasch überfordern. ${ }^{63}$ Für die Bundesrepublik Deutschland müssten zum Beispiel neben den 16 Bundestagswahlen insgesamt 199 Landtagswahlen ausgewertet werden, die im Zeitraum von 1946 bis heute (Juni 2009) stattgefunden haben. Während Eckhard Jesse ${ }^{64}$ die Koalitionssignale auf Bundesebene in einem Aufsatz kürzlich erstmals zusammenfassend dargestellt hat, wurde das Thema auf Landesebene bisher nur in Einzelanalysen im Kontext der allgemeinen Koalitionsbildung beziehungsweise des Regierens in

62 Die Lösung liegt allerdings nicht in dem oft geforderten Übergang zur relativen Mehrheit, da auch diese nur funktionieren würde, wenn das Regierungslager im Bundesrat über mehr Stimmen verfügt als die Opposition. Zur Kritik und für einen alternativen Vorschlag, vgl. Frank Decker, Relative statt absolute Mehrheit? Zur Diskussion um die Abstimmungsregeln im Bundesrat, in: Recht und Politik, 44. Jg. (2008), H. 4, S. 213 - 216.

63 Vgl. Franz Urban Pappi / Alexander Herzog / RalfSchmitt, a.a.O. (Fn. 16), S. 495 f.

64 Eckhard Jesse, a.a.O. (Fn. 12). 
Koalitionen behandelt. ${ }^{65}$ Ein Forscher oder eine Forschergruppe, die sich um die Erlangung und systematische Auswertung der Daten bemühen, könnten hier also richtige Pionierarbeit leisten.

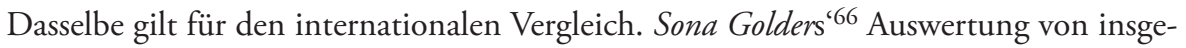
samt 339 Wahlen in 22 Demokratien von 1946 bis 1998 stützt sich auf Wahlanalysen und Fallstudien für die einzelnen Länder, in denen die Koalitionsaussagen aber oftmals nur am Rande betrachtet werden. Aufgrund der Lückenhaftigkeit der Daten sind die von ihr ermittelten Zahlen deshalb mit Vorsicht zu genießen, zumal sie von zuvor erschienenen Studien stark abweichen. ${ }^{67}$ Die Operationalisierung lässt ebenfalls zu wünschen übrig. Golder klammert zum Beispiel die negativen Koalitionssignale aus der Betrachtung aus und nimmt keine Unterscheidung zwischen starken und schwachen Signalen vor. Überwunden werden könnten diese Schwächen nur im Rahmen eines größeren Forschungsprojekts oder -verbunds, in dem die Länder von jeweils einem Experten (oder einer Expertengruppe) bearbeitet werden. Nachdem für die Koalitionsbildung in der Vergangenheit mehrere solche Projekte aufgelegt worden sind ${ }^{68}$, sollte das auch für die Koalitionsaussagen möglich sein.

65 Vgl. Uwe Jun, a.a.O. (Fn. 22); Sabine Kropp, Regieren in Koalitionen. Handlungsmuster und Entscheidungsbildung in deutschen Länderregierungen, Wiesbaden 2001.

66 Sona Nadenichek Golder, a.a.O. (Fn. 11).

67 Vgl. Lanny W. Martin / Randolph T. Stevenson, Government Formation in Parliamentary Democracies, in: American Journal of Political Science, 45. Jg. (2001), H. 1, S. 33 - 50.

68 Vgl. Wolfgang C. Müller / Kaare Strøm (Hrsg.), Coalition Governments in Western Europe, Oxford 2000 . 